\title{
Perivascular Spaces: Normal and Giant
}

\author{
Randy Fanous, Mehran Midia
}

\begin{abstract}
The purpose of this paper is to discuss both normal perivascular spaces (PVSs) and pathological giant perivascular spaces (GPVSs). The anatomy and physiology of normal PVSs, including important immunological and lymphatic roles, are described. Special attention is given to the Magnetic Resonance Imaging (MRI) findings of both normal and GPVSs. Furthermore, the clinical features and pathogenesis of GPVSs are explored, with special emphasis on the pathological implications of these lesions, and their relevance. It is important that symptomatic GPVSs not be mistaken for more devastating disease processes. When the lesions in question occur in a characteristic location along the path of a penetrating vessel, are isointense with cerebrospinal fluid on all MRI sequences, do not enhance with contrast material, are not calcified, and have normal adjacent brain parenchyma, their appearance is pathognomonic of GPVSs. The clinician should realize that an extensive differential diagnosis is superfluous and that biopsy is unnecessary in these patients. Instead, the clinical focus should be aimed at neurosurgical intervention, as dictated by the symptoms of mass effect.
\end{abstract}

RÉSUMÉ: Espaces périvasculaires normaux et géants. Le but de cet article est de discuter des espaces périvasculaires normaux (EPVNs) et des espaces périvasculaires géants (EPVGs). Nous décrivons l'anatomie et la physiologie des EPVNs ainsi que leur fonction immunologique et lymphatique qui sont des fonctions importantes. Nous portons une attention spéciale aux caractéristiques des EPVNs et des EPVGs à l'imagerie par résonance magnétique (IRM). De plus, nous examinons les manifestations cliniques et la pathogenèse des EPVGs, en soulignant les implications pathologiques de ces lésions et leur pertinence. Il est important de ne pas confondre des EPVGs symptomatiques avec des processus morbides plus dévastateurs. Quand les lésions en question surviennent à un endroit caractéristique le long du trajet d'un vaisseau pénétrant, sont isointenses par rapport au LCR sur toutes les séquences de l'IRM, ne sont pas rehaussées par la substance de contraste, ne sont pas calcifiées et que le parenchyme cérébral adjacent est normal, leur aspect est pathognomonique d'EPVGs. Le clinicien devrait réaliser qu'il est superflu d'envisager un diagnostic différentiel exhaustif et qu'une biopsie n'est pas nécessaire chez ces patients. Au point de vue clinique, l'attention devrait au contraire se porter sur l'intervention neurochirurgicale, si le patient présente des symptômes d'effet de masse.

Can. J. Neurol. Sci. 2007; 34: 5-10

\section{Perivascular Spaces}

\section{Anatomy}

Perivascular spaces (PVSs), also referred to as VirchowRobin spaces, are interstitial fluid (ISF)-filled channels that house penetrating arteries, and emerging veins, as they enter and exit the cerebral cortex.

Perivascular spaces are extensions of the subpial spaces, and are in direct continuity with it. ${ }^{1-4}$ The subpial spaces are located between the brain parenchyma and the pia. The pia is a highly vascular membrane that intimately envelopes the brain and spinal cord. It is composed of a single layer of cells joined by desmosomes and gap junctions. This layer separates the subarachnoid spaces from the subpial spaces. It also harbors blood vessels along the surface of the brain before they enter, or after they exit, the brain parenchyma. The pia lines the PVSs superiorly (outside the cortex parenchyma), whereas the basement membrane of the glial limitans lines the PVSs peripherally (inside the cortex parenchyma). Within the PVSs are blood vessels, which are surrounded by perivascular macrophages and cells of fibroblastic origin. ${ }^{1,2}$ The PVSs extend around the entire vascular tree within the cortex, until the level of the capillaries. At this point, the vascular and glial membranes fuse together, obliterating the space between them ${ }^{1}$ (Figure 1).

\footnotetext{
From the Department of Radiology, McMaster University, Hamilton, Ontario, Canada. Received MAy 3, 2006. AcCEPTED In FINAL FORM SEPTEMBer 27, 2006. Reprint requests to: Randy Fanous, 802 Upper Kenilworth Avenue, Hamilton, Ontario, L8T 5A4, Canada.
} 


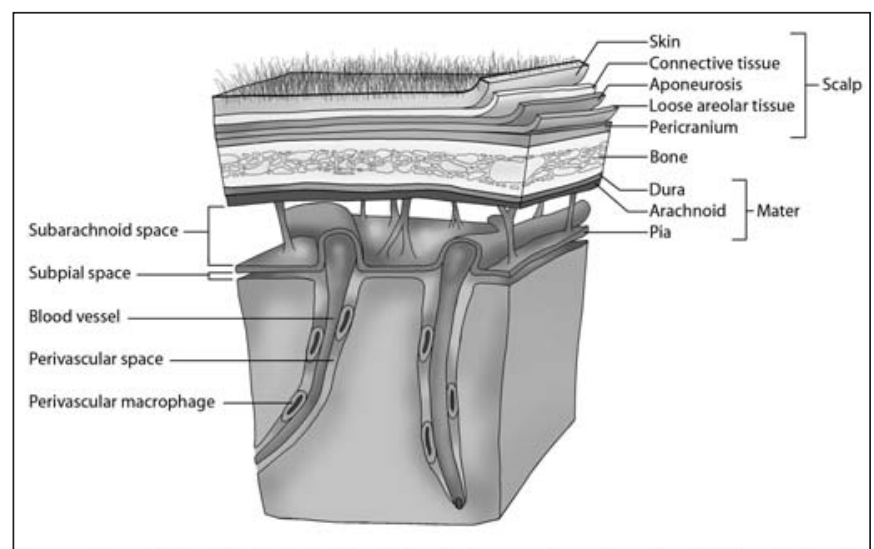

Figure 1: Coronal illustration depicting the relationship between the perivascular, subpial and subarachnoid spaces. Note that the pia intimately envelopes the brain parenchyma, separating the subarachnoid and subpial spaces. Note also that the subpial and perivascular spaces are in continuity.

The subpial and PVSs are filled with ISF, whereas the subarachnoid spaces are filled with cerebral spinal fluid (CSF). These two anatomical compartments are separated by the pia mater, yet can communicate. ${ }^{1,5}$ Studies have shown that tracer substances injected into the cerebral ventricles (and therefore the subarachnoid spaces) can be found in the PVSs within minutes. ${ }^{1}$

\section{Physiology}

Although not fully understood, it is believed that PVSs play important lymphatic and immunological roles. Perivascular spaces may provide a site for local immunological reactions to occur. In animals free of experimental manipulation and humans free of neurological disease, immunocytochemical studies have confirmed the presence of macrophages in PVSs. These findings suggest that a resident population of macrophages normally exists in the PVSs comparable to those lining the subarachnoid space. ${ }^{1}$ Using macrophage-specific monoclonal antibodies, studies have confirmed that these perivascular macrophages differ from resident brain microglia. ${ }^{1}$

Perivascular spaces act as compartments that are in communication with lymphatics of the head and neck, draining both ISF and CSF. ${ }^{1,3}$ Although experimental studies suggest that the main drainage route of ISF into the lymphatics is via the PVSs, this is not the case for CSF. The vast majority of CSF lymphatic drainage occurs through the blood via the arachnoid villi. Nevertheless, it is believed that ISF and CSF may reach the cervical lymphatics by way of leptomeningeal sheaths accompanying cranial and spinal nerves, as well as though communications between PVSs and lymphatic channels in the walls of major cerebral arteries. ${ }^{1}$

Drainage of ISF and CSF into the lymphatic system provides an opportunity for foreign antigens to be phagocytized by the perivascular and leptomeningeal macrophages. Phagocytized antigens are processed locally or conveyed to lymph nodes for presentation to helper $\mathrm{T}$ cells and $\mathrm{B}$ cells via MHC class II molecules. Once activated, these cells may accumulate in PVSs and generate an immune response to foreign antigens in the brain. ${ }^{2,6}$ The role of PVSs in immune defense also suggests a possible autoimmune basis for neurological diseases, such as encephalitis and multiple sclerosis. ${ }^{1,4}$

\section{Location}

Perivascular spaces may be both numerous and widespread throughout the brain, and are characteristically asymmetric. ${ }^{7,8}$ The most common location of PVSs is along the lenticulostriate arteries, just above the anterior perforated substance and adjacent to the anterior commissure 9,10 (Figure 2). Other common locations include the midbrain, subcortical white matter, and the subinsular cortex. ${ }^{6}$ Less common locations include the thalami, dentate nuclei, corpus callosum, cingulate gyrus, and the cortical white matter. ${ }^{3,11}$ However, with advancing age PVSs can be seen almost anywhere in the brain parenchyma. ${ }^{11}$

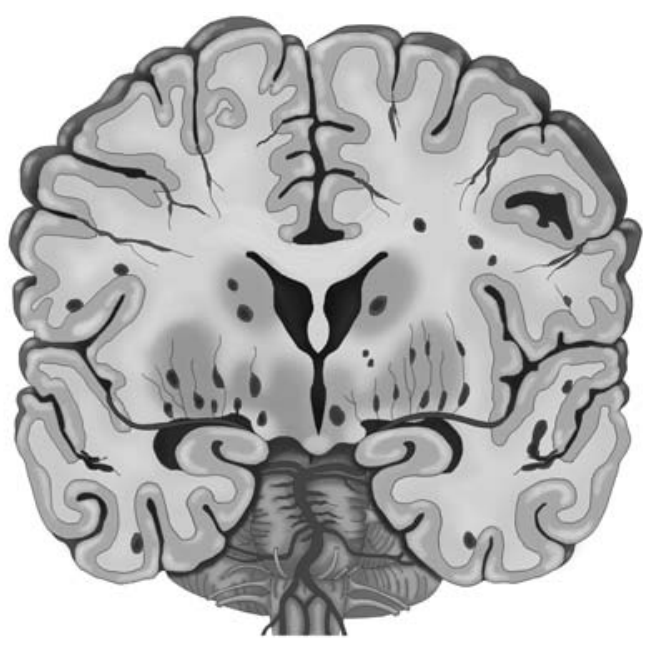

Figure 2: Coronal illustration depicting normal PVSs along the lenticulostriate arteries that penetrate the basal ganglia.

\section{Imaging}

With the advent of high-spatial-resolution MRI, it is possible to visualize normal PVSs in the brain. T2-weighted MRI is known to detail fine structures in and around the CSF spaces, and is considered a sensitive method for delineating such lesions. ${ }^{3,5}$

Typically, PVSs are seen on MR images as small foci $(<2$ $\mathrm{mm}$ in diameter) that are round or oval with a well-defined, smooth margin. They occur along the path of penetrating arteries, and are isointense with CSF on all pulse sequences. ${ }^{2,6,9}$ Furthermore, PVSs do not demonstrate enhancement following contrast administration, and do not show any other abnormalities, such as calcification, or signal changes of adjacent brain parenchyma., 

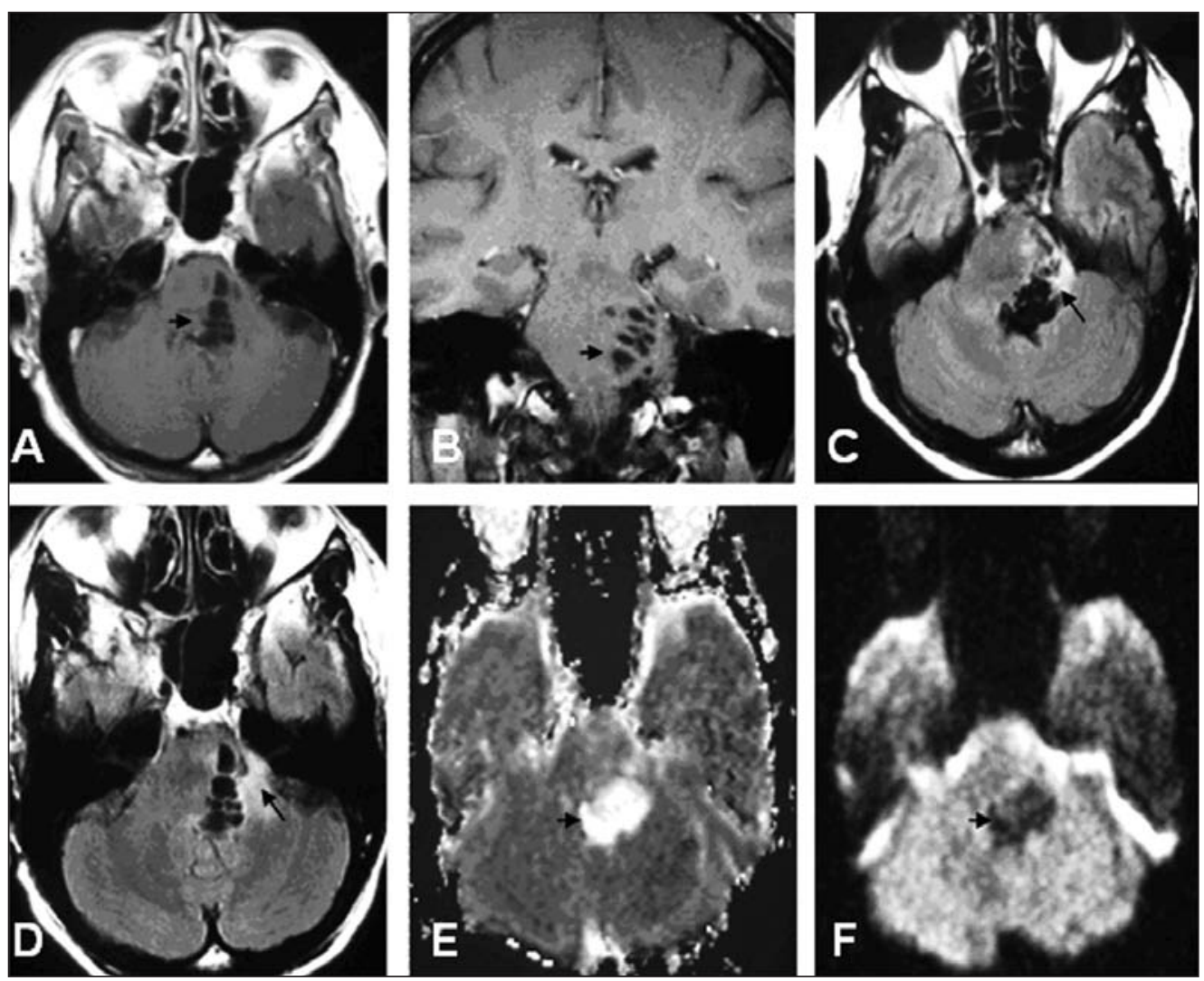

Figure 3: Magnetic resonance images obtained from a 50-year-old female with a chief complaint of headache. The MRI findings are in keeping with GPVSs. (A) Axial post-contrast T1-weighted image shows a septated cystic mass within the left side of the pons with some mass effect (arrow). (B) Coronal post contrast T1weighted fat-saturated image shows no enhancement associated with the lesion. Note the stacked-like appearance of the septations (arrow), corresponding to the course of the corticospinal tract in the pons. $(C, D)$ Axial FLAIR images of the basal- and mid-pons, respectively. Note the area of hyperintense signal alteration along the anterolateral aspect of the lesion (slanted arrows). Although not common, this finding has been described with GPVSs. Apparent diffusion coefficient map $(E)$ and diffusion-weighted $(F)$ images show no diffusion restriction. Similar to conventional series, the lesion (arrows) is isointense with CSF on all sequences.

\section{Prevalence}

Previous investigations have described small $(<2 \mathrm{~mm})$ PVSs as anatomical variants of normal found in up to $13 \%$ of adults. ${ }^{7,10}$ However in a recent study, Inglese et $\mathrm{al}^{2}$ reported a prevalence of up to $76 \%$. This difference has been attributed to variation in the spin echo sequence used, and the thickness of MRI slices obtained. Further studies are needed to delineate a more accurate prevalence of normal PVSs.

\section{Giant Perivascular Spaces}

\section{Morphology}

Perivascular spaces can expand and become enlarged. The extreme form of enlarged perivascular spaces are known as tumefactive or giant PVSs (GPVSs). Historically, GPVSs have also been referred to in the literature as etat crible (cribriform state), cavernous dilatations and Poirier's Type IIIb expanding lacunae.
Previous authors have defined GPVSs as greater than or equal to $1.5 \mathrm{~cm}$ in size. These entities are round, ovoid or linear in shape. They may occur as either a large solitary parenchymal cyst (Figure 3), or as a cluster of multiple, contiguous, welldemarcated parenchymal cysts of variable size (Figure 4). According to Salzman et $\mathrm{al}^{3}$ the later is more common.

\section{Location}

Giant perivascular spaces are found almost anywhere in the brain parenchyma, with the exception of the cortex where they rarely occur. Contrary to normal PVSs, the most common location of GPVSs is in the midbrain. ${ }^{3}$ The reason for this remains obscure.

In a sample of 115 patients with neurological complaints without cerebral disease, MRI revealed an incidence as high as $87 \%$ for GPVSs at the ponto-mesencephalic junction (PMJ) and an incidence as high as $63 \%$ at the mesencephalo-diencephalic 


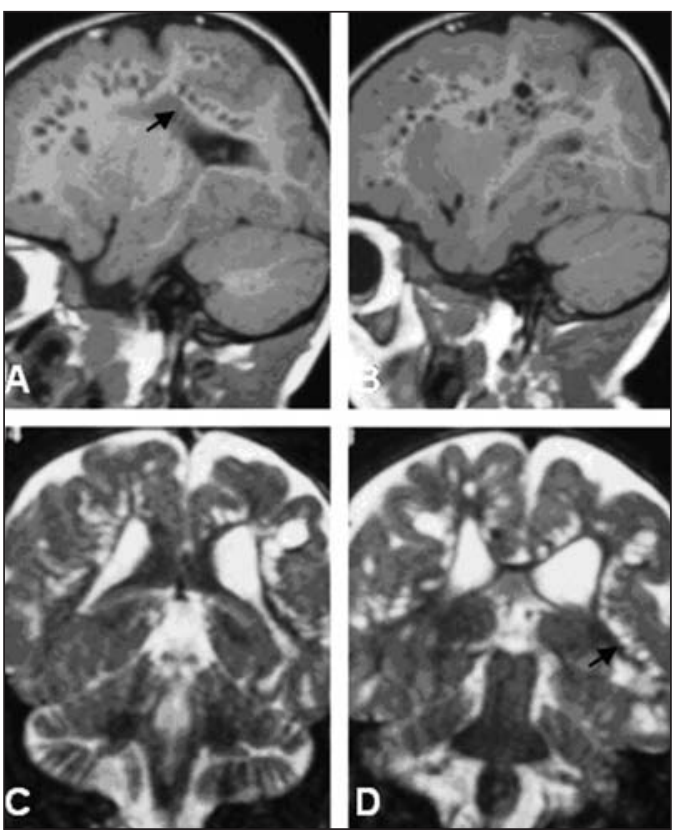

Figure 4: Magnetic resonance images obtained from an 8-year-old boy with Hurler syndrome. The MRI findings are suggestive of diffuse dilated PVSs. Sagittal T1weighted $(A, B)$ and coronal T2-weighted $(C, D)$ images show multiple linear foci, isointense with CSF, throughout the cerebral white matter (arrow). Note is also made of the underlying brain atrophy. junction (MDJ). This study conducted by Saeki et al supports the hypothesis that normal PVSs in the PMJ and MDJ may undergo pathologic modifications and expand, thereby causing morbidity. ${ }^{12}$ These findings are also supported by the observation that PVS expansion most commonly occurs in the mesencephalothalamic region in the territory of the paramedial mesencephalothalamic artery. ${ }^{3}$

\section{Clinical Features}

Giant perivascular spaces are usually incidental findings, discovered either during neuroimaging or at autopsy. However, patients with GPVSs may present with non-specific symptoms. According to Salzman et $\mathrm{al}^{3}$ the most common presenting symptom is headache. Other presenting symptoms include: dizziness, dementia, visual changes, seizure, syncope, stroke, memory problems, poor balance, and poor concentration.

\section{Imaging}

Once again, MRI is the modality of choice in diagnosing PVSs, whether normal or giant. On MRI, GPVSs are isointense with CSF on all sequences. They do not enhance with contrast, and are not calcified. Grossly they appear as either large solitary cyst-like lesions, or more commonly as multiple small contiguous ones of various size. Giant perivascular spaces occur in characteristic locations, along the path of penetrating vessels, and usually have normal adjacent brain parenchyma. ${ }^{3,12}$ Often, the penetrating vessel may be visualized clearly when contrast is administered.

In some cases, abnormal signal hyperintensity surrounding GPVSs are observed on T2-weighted MR images. This most often occurs in elderly patients, and may represent chronic small vessel ischemic changes or chronic mechanical stress on arterioles from hypertension. Less frequently in younger patients, more diffuse confluent areas of hyperintensity are observed, which may be attributed to gliosis or spongiosis, or may represent tightly clustered minute PVSs that are too small to be discriminated by MRI. ${ }^{3}$

Although MRI is the modality of choice, GPVSs are also seen on Computed Tomography (CT). On CT, GPVSs appear as clusters of non-enhancing cyst-like lesions of low attenuation.

Lastly, imaging should always be interpreted in the context of the clinical picture. It is important for the clinician to consider age, immune status, family history, and the presence of systemic illnesses. These details provide valuable clues that undoubtedly aid in the correct diagnosis of these lesions.

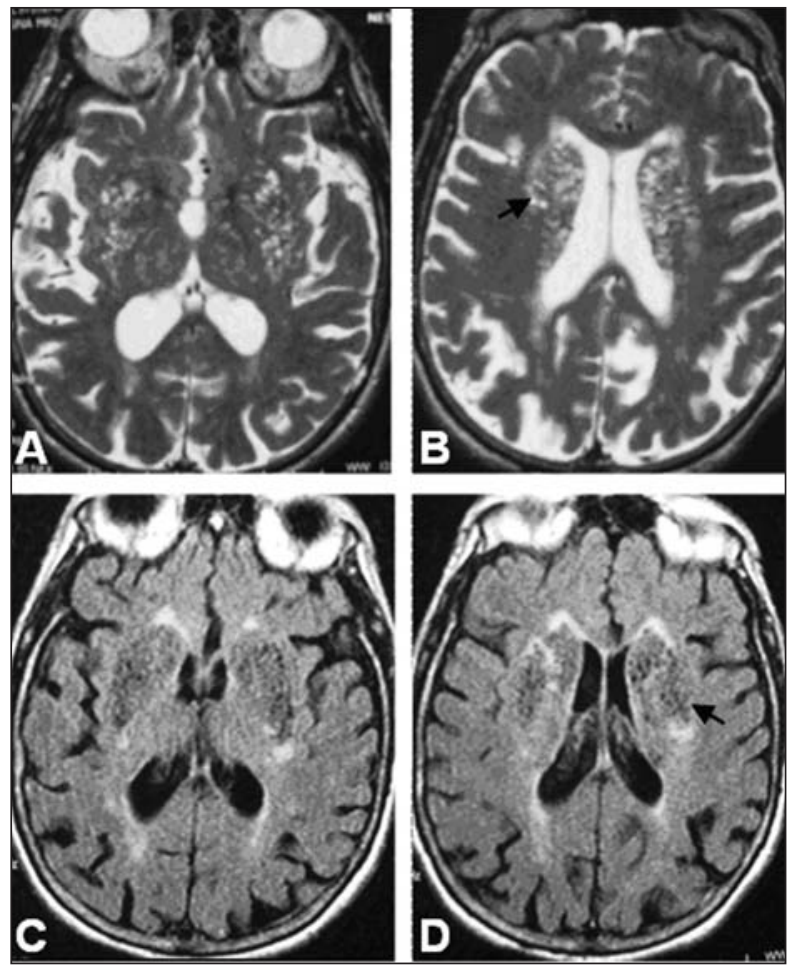

Figure 5: Magnetic resonance images obtained from a 65-yearold elderly female. Axial T2-weighted $(A-B)$ and axial FLAIR $(C$ $D)$ images show innumerable punctate foci that are isointense with CSF (arrows). These lesions are located in the putamen and lentiform nuclei bilaterally. This is consistent with dilated PVSs of the perforator branches that arise from the anterior and middle cerebral arteries. Minimal gliosis is also noted surrounding the basal ganglia in $(C)$ and $(D)$. 


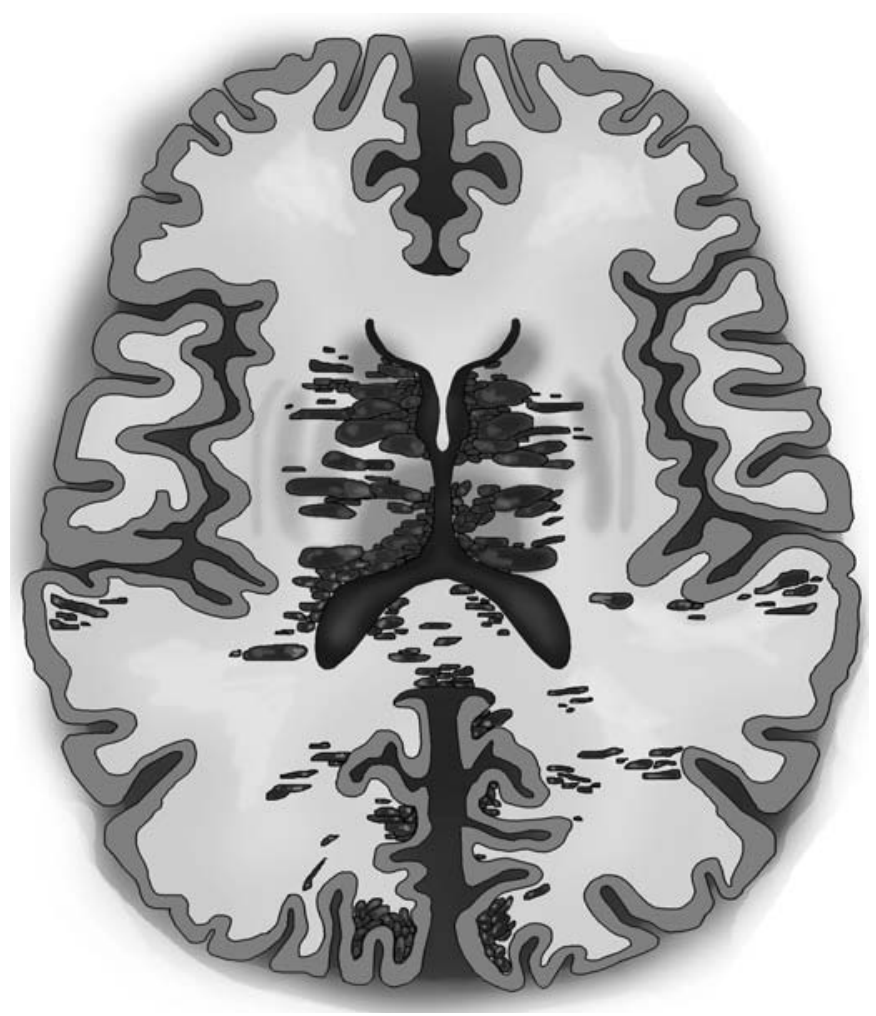

Figure 6: Axial illustration depicting GPVSs in the basal ganglia and white matter. Note that the periventricular pattern of disease seen in this artistic representation is similar to that of the T2-weighted MR images in Figure $5(A, B)$.

\section{Pathogenesis and Pathological Implications}

The precise pathological processes leading to dilatation of the PVSs remains obscure; however, many possible mechanisms have been suggested in the literature. Three main hypotheses have been introduced: a) increased arterial wall permeability due to vasculitis, b) impaired ISF drainage into ventricles due to increased intraventricular CSF pressures, and c) impaired ISF drainage due to lymphatic obstruction. ${ }^{3,4,6,8}$ Other possibilities include inflammatory destruction (by perivascular free radicals and cytokines), brain atrophy, ${ }^{2}$ and spiral elongation of penetrating blood vessels due to systemic hypertension. ${ }^{13}$

A retrospective study conducted by Heier et $\mathrm{al}^{10}$ compared two groups of subjects, with and without expanding PVSs, for age, gender, white matter lesions (WMLs), infarction, dementia, hypertension and atrophy. Positive associations with enlarged PVSs were found with age, hypertension, dementia and WMLs. However, after logistic regression analysis, only age remained significantly associated with the presence of GPVSs (Figures 5 and 6). Nevertheless, it is important to note that these results conflict with more recent data by other groups that deny a relationship between the number of PVSs and age. ${ }^{9}$

MacLullich et $\mathrm{al}^{14}$ tested the hypothesis that GPVSs are associated with poorer cognitive function in a sample of healthy elderly men. The authors concluded that GPVSs are correlated with worse cognitive function. However, when controlled for the coexistence of WMLs, GPVSs did not seem to have an independent contribution to decreased cognitive function. ${ }^{11}$

Recently, Inglese et al have explored the relationship between mild traumatic brain injury (TBI) and GPVSs. Although MRI shows no abnormality in most cases of mild TBI, $30 \%$ of these patients have some degree of neurological or cognitive deficit. According to the authors, structural and physiological factors most likely contribute to the deficit in these patients. The number of GPVSs in 24 patients who suffered mild TBI was compared to 17 age- and sex-matched healthy control subjects. The authors found that the mean number of GPVSs was significantly higher in patients than in control. ${ }^{2}$ However, the study lacked baseline MR assessments and cognitive testing prior to injury. Therefore, the possibility that the number of GPVSs was increased in the patients prior to trauma cannot be ruled out. ${ }^{2}$

In a second study by Inglese et al, 38 patients with mild TBI, and 21 controls, were assessed with MRI and 13 cognitive tests. Fifteen subjects underwent MRI within a mean interval of 5.4 (112) days, and the remaining 23 subjects after a mean interval of $5.5(0.2-31)$ years. The average number of PVSs visualized on T2-weighted MR images was significantly higher in patients (6.9 $\pm 4.3)$ than in controls $(3.0, \pm 4.0)$ yielding a p value of 0.002 . However, this was not directly associated with cognitive function. Therefore, the authors concluded that in mild TBI, enlarged PVSs likely represent irreversible morphological changes secondary to shear-strain, but do not have functional implications. Similar to the diffuse axonal injury (DAI) of neurons that occurs in TBI, penetrating blood vessels may also be injured by shear-strain. This may in turn lead to permanently dilated PVSs. In addition, the average number of PVSs did not differ between patients within days or years of injury. This suggests that GPVSs represent a permanent morphological change that do not significantly progress or regress. ${ }^{9}$

From these and other studies, it is clear that further investigations are warranted in order to determine whether GPVSs are pathological manifestations, or a benign manifestation of aging. ${ }^{2}$

\section{Relevance}

It is clear that PVSs become enlarged and more numerous in some disease states $^{9}$ (Table 1). Likewise, it is also clear that GPVSs are not viable tissue, and thus represent a mild form of local atrophy. ${ }^{11}$ What remains unclear however, is the precise prognostic value of these lesions. Nevertheless, the clinical significance must not be overestimated by the clinician.

Small PVSs have been identified on MR images of all age groups, ${ }^{6,7}$ in both symptomatic and asymptomatic patients. Small PVSs do not typically result in morbidity since they lack mass effect, and are seen as a variant of normal. However, when the PVSs expand to become GPVSs, they can act as space occupying lesions, and cause pathology. 6,12

Giant perivascular spaces are rare lesions on MRI that can mimic findings of vascular dementia such as leukoaraiosis or etat crible (cribriform state), obstructive hydrocephalus, ${ }^{3,6}$ cystic tumors, ${ }^{8}$ human immunodeficiency virus encephalopathy, ${ }^{1}$ multiple sclerosis, ${ }^{1}$ fungal Cryptococcus neoformans infection, ${ }^{6}$ 


\section{Table 1: Disease states associated with GPVSs}

\section{- Aging}

- Dementia

- Traumatic brain injury

- Obstructive hydrocephalus

- Epilepsy ${ }^{16}$

- Multiple sclerosis

- Neurosarcoidosis

- Mucopolysaccharidoses (Hurler, Scheie, Hurler-Scheie syndrome

- Sener syndrome

- Parkinson's disease

- Encephalitis

- Human immunodeficiency virus encephalopathy

- Fungal Cryptococcus neoformans infection

- Cerebral arteriosclerosis

- Cerebral autosomal dominant arteriopathy with subcortical infarcts and leukoencephalopathy (CADASIL)

- Coffin-Lowry syndrome ${ }^{17}$

- Hypertension

Diabetes

neurosarcoidosis, ${ }^{15}$ Sener's syndrome, ${ }^{4}$ and mucopolysaccharidosis ${ }^{4}$ among others. It is important that symptomatic GPVSs not be mistaken for more devastating disease processes. As highlighted by Salzman et $\mathrm{al}^{3}$ when the lesions in question occur in a characteristic location along the path of a penetrating vessel, are isointense with CSF on all MRI sequences, do not enhance with contrast material, are not calcified, and have normal adjacent brain parenchyma, their appearance is pathognomonic for GPVSs $s^{3,12}$ (Table 2). Therefore, the clinician should realize that an extensive differential diagnosis is superfluous and that biopsy is unnecessary. ${ }^{3}$ Instead, the clinical focus should be aimed at neurosurgical intervention, as dictated by the symptoms of mass effect. ${ }^{8}$

\section{Table 2: MRI characteristics pathognomonic of GPVSs}

- Location along path of a penetrating vessel

- Isointense with CSF on all MRI sequences

- Do not enhance with contrast material

- No calcifications

- Normal adjacent brain parenchyma

\section{REFERENCES}

1. Esiri MM, Gay D. Immunological and neuropathological significance of the Virchow-Robin space. Neurol Sci. 1999; 100(1-2):3-8.

2. Inglese M, Bomsztyk E, Gonen O, Mannon LJ, Grossman RI, Rusinek H. Dilated perivascular spaces: hallmarks of mild traumatic brain injury. Am J Neuroradiol. 2005; 26:719-24.

3. Salzman K, Osborn AG, House P. Giant tumefactive perivascular spaces. Am J Neuroradiol. 2005; 26:298-305.

4. Lynch SA, Hall K, Precious S, Wilkie AOM, Hurst JA. Two further cases of Sener syndrome: frontonasal dysplasia and dilated Virchow-Robin spaces. J Med Genet. 2000; 37:466-70.

5. Saeki N, Nagai Y, Matsuura I, Uchino Y, Kubota M, Murai H, et al. Histologic characteristics of normal perivascular spaces along the optic tract: new pathogenetic mechanism for edema in tumors in the pituitary region. Am J Neuroradiol. 2004; 25: 1218-22.

6. Papayannis CE, Saidon P, Rugilo CA, Hess D, Rodriguez G, Sica REP, et al. Expanding Virchow Robin spaces in the midbrain causing hydrocephalus. Am J Neuroradiol. 2003; 24:1399-1403.

7. Rollins NK, Deline C, Morris MC. Prevalence and clinical significance of dilated Virchow-Robin spaces in childhood. Radiology. 1993; 189:53-7.

8. Rohlfs J, Riegel T, Khalil M, Iwinska-Zelder J, Mennel HD, Bertalanffy $\mathrm{H}$, et al. Enlarged perivascular spaces mimicking multicystic brain tumor. J Neurosurg. 2005; 102:1142-46.

9. Inglese M, Grossman RI, Diller L, Babb JS, Gonen O, Silver JM, et al. Clinical significance of dilated Virchow-Robin spaces in mild traumatic brain injury. Brain Injury. 2006; 20(1):15-21.

10. Heier LA, Bauer CJ, Schwartz L, Zimmerman RD, Morgello S, Deck MD. Large Virchow-Robin spaces: MR-clinical correlation. Am J Neuroradiol. 1989; 10:929-36.

11. Barkhof F. Enlarged Virchow-Robin spaces: do they matter? J Neurol Neurosurg Psychiatry. 2004; 75:1516-17.

12. Saeki N, Sato M, Kubota M, Uchino Y, Murai H, Nagai Y, et al. MR imaging of normal perivascular space expansion at midbrain. Am J Neuroradiol. 2005; 26:566-71.

13. Sawada M, Nishi S, Hashimoto N. Unilateral appearance of markedly dilated Virchow-Robin spaces. Clin Radiol. 1999; 54:334-6.

14. MacLullich AMJ, Wardlaw JM, Ferguson KJ, Starr JM, Seckl JR, Deary IJ. Enlarged perivascular spaces are associated with cognitive function in healthy elderly men. J Neurol Neurosurg Psychiatry. 2004; 75:1519-23.

15. Mirfakhraee M, Crofford MJ, Guinto FC, Nauta HJ, Weedn VW. Virchow-Robin space: a path of spread in neurosarcoidosis. Radiology. 1986; 158:715-20.

16. Bastos AC, Andermann F, Melancon D, Cindes F, Guberman A, Dubeau F, et al. Late-onset temporal lobe epilepsy and dilatation of the hippocampal sulcus by an enlarged Virchow-Robin space. Neurology. 1998; 50(3):784-7.

17. Patlas M, Joseph A, Cohen JE, Gomori JM. MRI and MRS of Coffin-Lowry syndrome: a case report. Neurol Res. 2003; 25(3):285-6. 